\title{
The cellular senescence unification model and telomerase therapy: To treat all age-related diseases
}

\author{
Steve Liebich ${ }^{\mathrm{a},{ }^{*}}$ \\ ${ }^{a}$ Department of Biomolecular Science \& Chemistry, Clarkson University, 10 Clarkson Ave, Potsdam, NY 13676, USA.
}

\begin{abstract}
Since the discovery of the telomere by Hermann Muller and Barbara McClintock in years 1938-1940, a great progress has been made in molecular genetics and in the relatively new field, biogerontology. Almost 40 years have passed since the discovery of telomerase by Carol Greider and Elizabeth Blackburn (1984). Since those major discoveries, the scientific community has linked many naturally occurring ageing mechanisms in cells to the shortening of telomeres and the lack of telomerase activity in these cells. A great number of mutagens, radiation, and toxic chemicals negatively impact the length of telomeres with their truncation triggering a fatal cascade of events inside the cell which can lead to the state of senescence and eventually to cell death. Even though cellular and bodily ageing is a complex, multilevel, and highly orchestrated natural process happening at the subcellular level of all multicellular organisms, and a single known mechanism is extremely insufficient to explain all observed molecular and morphological changes, a unified cohesive theory of ageing must exist. The cellular senescence unification (CSU) model, combining the free-radical-mitochondrial and telomeric theories, helps establish a strengthened base for future therapies of all age-related disorders. If the CSU model is strong enough to explain and describe most of all the observed alterations in the ageing cell, a focused and deliberate therapy might be developed. This work introduces telomerase therapy as an efficient, highly effective, and clinically favored treatment for most age-related disorders, which can be elucidated by the CSU model. Gene therapy is the next natural step forward for biogerontology since the discovery of the telomere 80 years ago.
\end{abstract}

Keywords: Telomeres, telomerase therapy, cellular senescence, unified theory of ageing

\section{Introduction}

Each eukaryotic cell division leads to a gradual sequence loss at the chromosomal termini known as telomeres [1]. This so-called end replication problem (ERP) forms the basis of cellular senescence, along with a few other wellestablished biochemical processes disrupting the cellular homeostasis [2].

Telomeres are composed of repeated oligonucleotide sequences supported by a six-protein complex (shelterin) and the quaternary structure they form in vivo [3-5]. Thus, telomeres are the specific genomic protection device,

\footnotetext{
* Corresponding author: Steve Liebich

Mailing address: Department of Biomolecular Science \& Chemistry, Clarkson University, 10 Clarkson Ave, Potsdam, NY 13676, USA.

Email: liebicsf@clarkson.edu
}

Received: 07 June 2020 / Accepted: 18 Septemper 2020 guarding the cell from the inexpedient recombination events, degradation through the DNA repair system, and significant genetic material loss [6-9]. In some cell types, a reverse transcriptase known as telomerase reconstitutes the original length of the shortening chromosomes, saving the chromosomal and genomic stability of the cell [10-14]. In such conditions, the cell undergoes almost an infinite number of mitotic cycles, breaking through the Hayflick phenomenon [15].

Cellular and bodily ageing result from complex, multilev$\mathrm{el}$, and precisely controlled mechanism of decline in effectiveness of physiological processes in the cell and decline in the stability of its genome. Because of this complexity, more than three hundred different or slightly different theories of ageing have been proposed [16]. Many the ories overlap, while the others leave gaps too broad to be neglected. New data are very often misleading and contradicting, thus supporting neither of the proposed rationales. Therefore, biogerontology needs to find a common denominator for all credible theories, so that one elegant unified theory could explain the entire life-long process of cellular ageing. 
All proposed ageing theories are segregated into two main categories: programmed and non-programmed [17]. The first category takes into account all ageing factors genetically inherited and gradually manifested over the span of a lifetime. The second group includes theories emerging from the customarily occurring errors in the genome and accumulated damage in the cell; tear-and-wear is preferably chosen as the classic theory considering accumulating damage in the genomic DNA. However, it had been the free-radical and mitochondrial theories that took much of the scientific community's appraisal in the last century [18-21]. Three decades ago, the telomeric theory of cellular ageing stole the spotlight and since has grown into a well-developed and data-supported biogerontological doctrine, which aims to explain most of the observed hallmarks of cellular senescence [22-25]. These two theories, the free radical-mitochondrial and telomeric, have the potential to form a single comprehensive model defining the complexity of ageing. It is referred to here as the cellular senescence unification (CSU) model. For clarity purposes and due to space limitation, only the telomeric theory will be introduced and described.Is telomerase, the enzyme of capacity to restore the shortened telomeres and stabilize the genome, the definitive answer to the puzzling problem of ageing? Could we take advantage of this finding and apply it to humans, hence aim to improve health, fight age-related diseases (ARDs), and even reverse the ageing machinery in their cells? This article elaborates on the subject and aims to address these two questions with proper and preservative analysis of recent advances in the field of biogerontology.

\section{The cellular senescence and ageing unification theory}

As Bodnar et al. showed, each human cell which does not express an active hTERT (human telomerase reverse transcriptase) transcript loses its terminal chromosome repeats [11]. If cellular senescence was viewed as a dense system of all cellular and genomic changes occurring over an undefined span of time, relative loss of telomeres would be both one of the causes and effects of the total intracellular changes. By now, telomere loss and lack of telomerase expression in ageing cells are some of the most studied phenomena in ageing biology. However, it is not, and it must not be treated as the cause of gradual cellular dysfunction. Since the paper of L. Hayflick and P. Moorhead on the limited mitotic capacity of somatic cells was published, the link between the restricted cell doublings and their mortality, telomere shortening and the inevitable replicative senescence phenomenon has become obvious [15]. A large number of publications have been dedicated to the association of telomere shortening with numerous agerelated disorders (see below).

A few aspects of cellular senescence must be considered before further discussion. Senescence is not equivalent to the quiescent state of the cell [26]; cell lines like hepa- tocytes and corneal endothelial cells maintain replicative capability, but do not divide without external stimuli [27, 28]. Cellular senescence is defined then as the permanent quiescence of the cell cycle. The all-or-nothing model of cellular senescence, popularized in the past century, has lost its merits over the more consistent and data-supported gradual changes within the cells [22]. Today, the cell senescence model of aging is acknowledged and the postulate that senescence and the non-programmed errors accumulated in genome result from changes in, among others, gene expression and the epigenetic landscape seems to be satisfying and unify other related theories [29].

Due to space limitation and deliberate focus on the telomeric theory, some programmed and non-programmed factors of cellular ageing further explained will be discussed in the light of telomeres shortening, however, those factors reach beyond the telomere biology and affect a plethora of other intracellular sites and processes. Recent research advances in biogerontology show a moderately strong correlation between telomere shortening and other possible processes contributing to cellular ageing, which all cumulatively (and definitely not singlehandedly) lead to the senescent state of the cell, which can be defined as a quiescent (non-mitotic) state of the cell unresponsive to the external stimulation and such cell is found in the G0S stage of the cell cycle. As discussed below, telomere shortening, chromatin destabilization, change in genome dynamics, uneven generation of free radicals, and alteration in gene expression patterning are unequivocally correlated, which may help to draw more definitive conclusions in the future (Figure 1).

After all, senescence is a gradual process occurring in every cell of a multicellular organism, including cells that are mitotically inactive. Senescence should be understood as a complex network of changes in genes expression, DNA damage, ineffective DNA repair mechanisms, misbalance between reactive oxidative species production and scavenging, cell morphology, toxins accumulations, proteomic changes, and finally loss of telomeres. The telomeres field has had over half a century of research and promising findings which in consequence makes it both one of the most studied cellular senescence biomarker and the target for potential gene therapy; the encouraging studies of re-lengthening the shortened telomeres in animals (in vivo and in vitro) and in humans (in vitro) only seal the inevitable importance of the telomeres role in ageing. Even though it is subjective and experimentally unapproachable to test whether a cell is already senescent or "not yet," the CSU model is supported by our current knowledge, and although not perfect, it is still the best tool we can use to not only explain cellular ageing processes, but also to manipulate them.

Fossel (2012) suggests the relative telomeres length measures as a reliable, clinically practical, and specific biomarker of age-related diseases [30]. Correlation between the telomere corrosion in a chronologically old individual and onset of various age-related diseases is well-known, thus telomeres and their shortening seem to be a reliable 


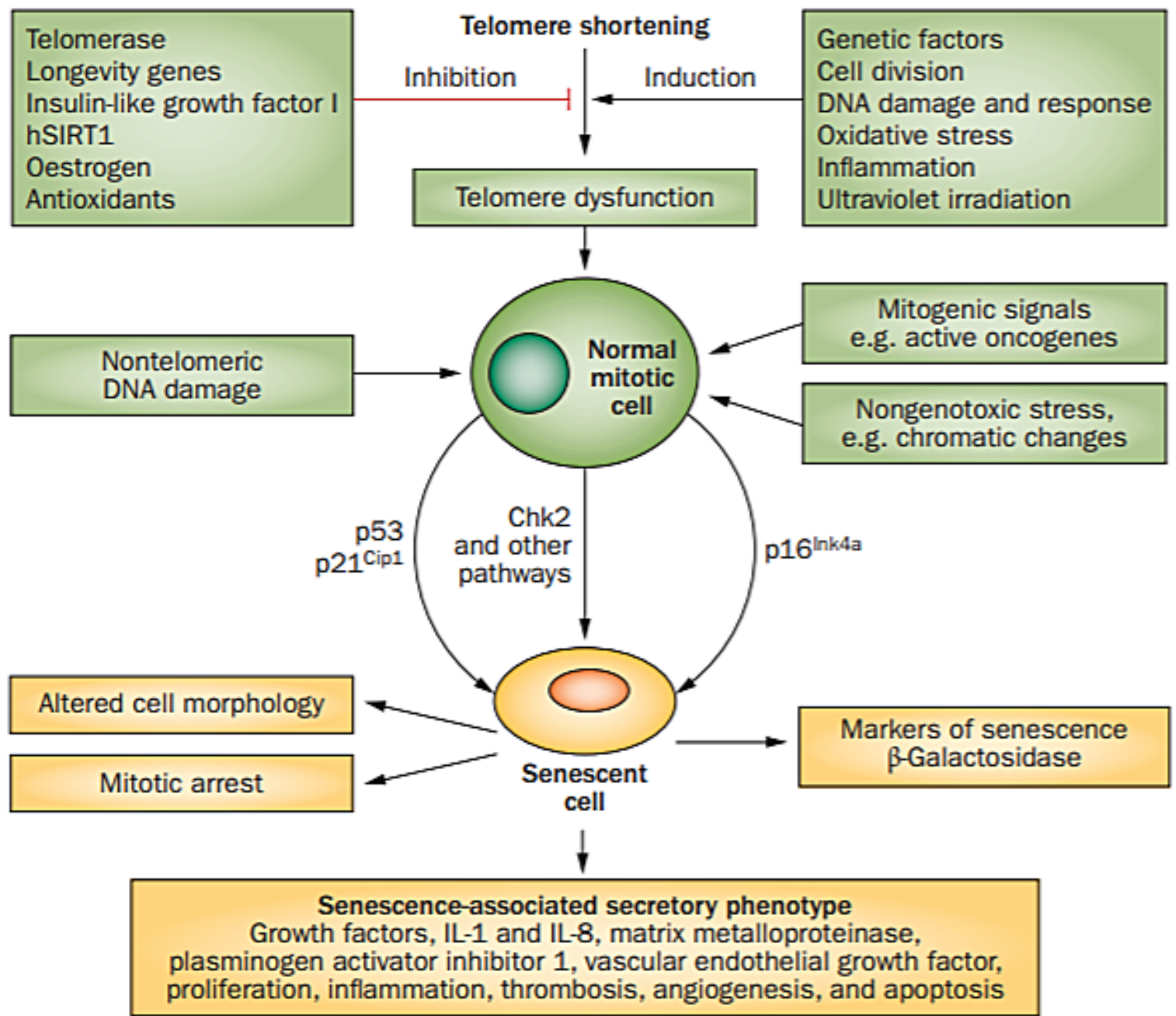

Figure 1. Cellular senescence: external and internal factors. A plethora of mutagens, signaling pathways, cytokines, and oxidative agents influence the rate of telomere shortening in the cell. Environmental stressors (UV light, industrial toxins, carcinogens, and intercalating agents), expressed oncogenes, and chromatin alterations also affect the progression of cellular senescence. Tumor suppressors, including p53, are activated upon those deleterious signals and respond adequately by triggering mitotic arrest, altering cell morphology, secretion of growth factors, cytokines, and apoptotic factors. Telomeres are affected by all aforementioned senescence factors, but the same factors induce their effects through other genomic and non-telomeric pathways. Abbreviations: hSIRT1 (NAD-dependent protein deacetylase sirtuin-1); Chk2 (checkpoint signaling kinase 2). Figure adapted from [51].

source of clinical information and a platform for proper intervention. It must be noted that it is the relative rate of telomeres shortening, not just a total loss of their sequences. This gives an insight into the onset of telomeredependent diseases and their clinical manifestation [31]. This remark has been taken to clinics with commercial enterprises of key telomere researchers: Maria Blasco (Life Length) and Calvin Harley \& Elizabeth Blackburn (Telome Health). These are only the first steps of the telomere research evolving into clinical importance.

However, as long as the relative telomere loss is a strong biomarker in chronologically advanced patients and individuals in greater risk cohorts, the very common telomere length (TL) measure in peripheral blood leukocytes (PBLs) seem to be an undesirable method. First, false negative results might affect the diagnosis of a patient still affected by slowly evolving pathological processes. The reason is that leukocyte telomeres might remain relatively stable if a disorder develops in the liver, for instance; moreover, the "old" leukocytes are constantly being replaced by the white blood cells with long telomeres. Second, peripheral leukocytes can be exposed to toxic, stressful or immunological factors that would influence telomere loss in the PBL without any underlying age-related disorder [32]. Multiple studies showed a positive correlation between external factors such as smoking, obesity [33], oxidative damage [34, 35] and past infectious diseases [36-38]. Third, the genomic changes of white blood cells (WBC) are just as important as in other human cells: the age-dependent telomere shortening [39], polymorphisms in the hTERT promoter and its regulatory genes [40] (although a Swedish cohort studies of the same single nucleotide polymorphism did not show any correlation [41], and changes in the epigenetic landscape of the hTERT [42]. These findings indicate that the relative PBL telomere loss is a significant biomarker for a number of age-related 
diseases, but PBLs are not always the right source of telomere attrition information.

Coronary heart disease [43], osteoporosis [44], diabetes [45], and other age-related disorders are correlated with shortened telomeres; the telomere attrition leads to cell senescence as observed in multiple tissues and organs. PBL telomere length (TL) measurements have been applied to a plethora of various age-related disorders, diseases of affluence and immunological disorders, including longitudinal studies of cardiovascular health problems [46], chronic obstructive pulmonary disease [47], familial and sporadic pulmonary fibrosis [48], and hematopoietic malignancies [49]. These data underlie a strong correlation between telomere attrition and the etiopathophysiology of diseases observed in clinical settings.

Human skin fibroblasts were the first telomere-associated senescence model cells, for which the telomerase transfection proved to be liable [11]. In two parallel studies, human keratinocytes and fibroblasts were grown on an immune-compromised mouse and the new skin morphology was assessed for early (20 population doublings) and late (85 population doublings) passages; the late passage cells were further transformed with an external hTERT and the re-lengthened telomeres led to skin reconstitution, optimal gene expression and normal filamentous connections observed in the young skin [50].

Almost every tissue type in the human body demonstrates histological and ultracellular changes associated with telomere shortening. For cardiovascular diseases, mice and human models show exceptional correlation between telomere dysfunction (also oxidative stress, proinflammatory molecules activation, etc.) and senescence of vascular endothelial cells leading to development of cardiomyopathy and severe atherosclerosis [51]. The impairment of control mechanisms of stem cell reserves and their differentiation and division in the bone marrow, hugely associated with telomere attrition and immune system age-related changes, is responsible for the pathological decrease in activity in older people. Similar deferment in physiology has been found in glial cells, the cells that divide and proliferate in the brain, whose ultracellular ageing transformation has been presented to be the leading cause of Alzheimer's disease [52]. These examples indicate the necessity to reform the way how one interprets the pathophysiology of many age-related disorders. The non-divisional pattern of neural or myocardial cells would lead to an inaccurate and misguided conclusion that the telomeric theory could not be taken into account for these cells. However, this inaccuracy stems from the wrong perspective: one must shift focus from the mitotically inactive to the dividing cells found in the proximity of the non-divisional cells. The combined pathophysiologic outputs of the non-dividing, but still homeostatically deteriorating cell and the mitotically active cell, whose telomeres do truncate after each round of mitosis (next to other countless ultracellular ageing processes) draw a more consistent and self-explanatory picture of age-related disorders.

\section{Control mechanisms of telomere length and telomerase expression}

Senescence is a gradual process that takes place in every living cell of the human body, with cells varying in degrees of senescence. This is a complex and global process incorporating changes in epigenetic status, genomic mutations and breakages, slowed metabolic turnover of the cell, increased levels of oxidative species, failing mitochondria, accumulation of toxic chemical compounds (external and internal), deterioration of signaling pathways, increased turnover of proteins, and increasing area to volume ratio of the cell.

Not all changes lead to the cell's death though. Senescent cells rarely "die," they rather move to a quiescent state where they become less or completely mitotically inactive. However, shortened telomeres in somatic cells advance genomic defects, and this destabilization leads to more rapid cellular changes $[23,25]$. On the other hand, stem cells are partially protected from the instability by expressing telomerase, an enzyme that extends the missing chromosomal termini. The difference between somatic and stem cells is that the former has an accelerated rate of senescence, while the latter gradually become more senescent $[53,54]$. Thereby with old age, stem cells are more senescent than they were in the young organism, but somatic cells are more senescent than the stem cell populations. While most organs store their somatic stem cells in so-called niches, which function as "spare parts" for the ageing organ, the process of replacing the aged and/or dead somatic cells with transit cells (dividing and slightly differentiating stem cells) demonstrating self-renewal capability diminishes [54-56].

Telomerase plays an important part in keeping the cell's homeostasis balanced. Therefore, telomerase expression and activity regulation in the cell is highly significant. The 40-kb hTERT locus sits on chromosome 5 , only $2 \mathrm{Mb}$ away from the chromosomal end [57]. hTERT lacks both TATA and CAAT boxes, but is regulated with two canonical E-box sequences [12] (Figure 2). The E-box sequences and numerous $\mathrm{CpG}$ islands provide large methylation sites for the hTERT locus, which demonstrates high epigenetic regulation [58]. Most human cells do not produce telomerase [12]. The exceptions are stem cells, germ cells, and select white blood cells $[59,60]$. Moreover, $85-90 \%$ of cancer cells express active telomerase [61-63]. It is still unknown why most somatic cells are hypermethylated at the telomerase site and what exact mechanisms participate in the negative regulation of the locus [64]. However, various findings show that some cells are capable of producing telomerase in vivo, but these are inactively spliced variants that do not contribute to the telomerase activity [65].

The telomerase-telomere cellular "apparatus" is also controlled by a wide network of proteins. The trans-acting regulatory proteins are extremely active at the hTERT locus. The most abundant transcription factor (TF) com- 


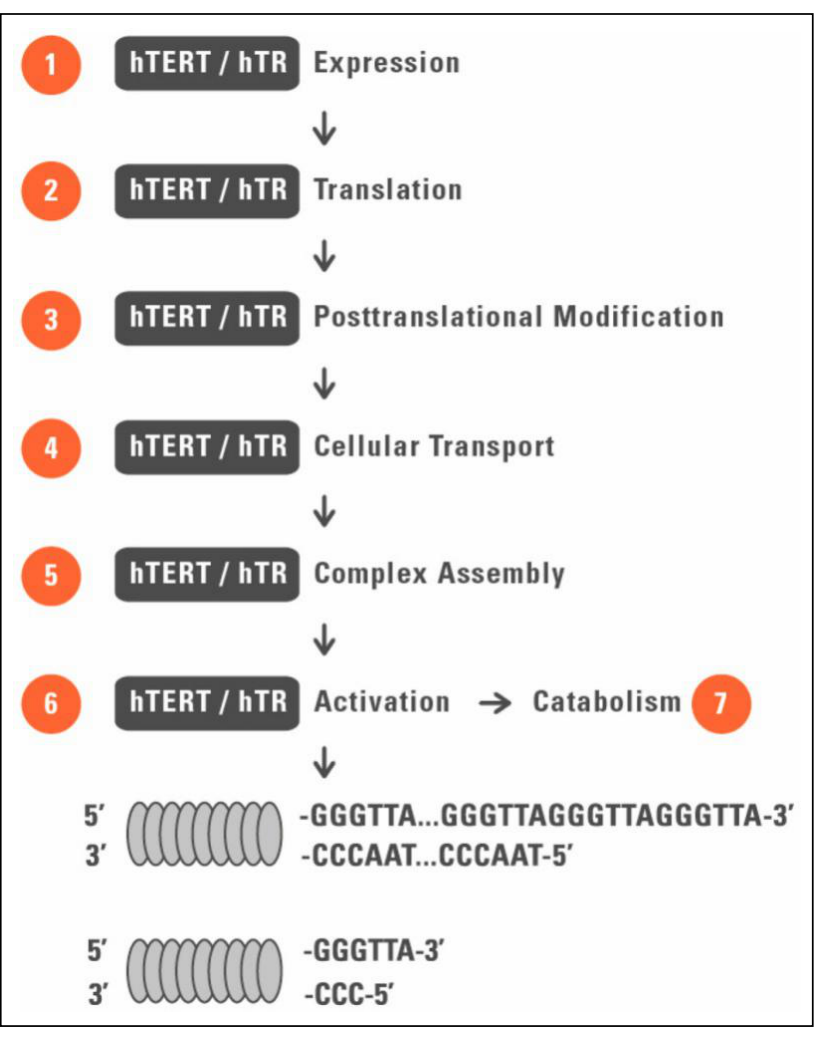

Figure 2. Telomerase expression mechanism in telomerase-positive cell line. Telomerase is a ribonucleoprotein complex composed of many subunits, of which telomerase reverse transcriptase (TERT) and telomerase RNA component (TERC) are essential in physiological activity of telomerase. The multicomponent enzymatic complex extends chromosomal termini by synthetizing de novo tandem telomeric repeats (5'TTAGGG-3') at 3' end of the chromosome. TERC binds the 5 $\mathrm{C}$-rich strand and its 11-nt sequence serves as the replication template for reverse transcription. Abbreviations: hTERT (human telomerase reverse transcriptase); hTR (human telomerase RNA component). Figure adapted from [115].

plexes are E-box-binding c-Myc/Max complex, which cooperates with the GC-box far-flung Sp1 TF acting as the activating element [66]; on the other hand, Mad1/Max complex, along with deacetylases, represses the hTERT locus [67]. To review the divergent transcription factors involved in the telomerase expression regulatory mechanisms refer to [68-70].

Telomeres are supported by another, but not exclusive, set of proteins. The already mentioned shelterin complex, composed of six core proteins, both protects telomeres from exonucleases degradation and provides an interaction matrix for other regulatory factors [71]. The telomeres instability is reflected mainly through the double-strand break (DSB) events. The DSBs induce chromosomal translocations, error-prone recombination events and tumorigenic processes [72]. Two major DNA damage sensing pathways are related to ATM and ATR serinethreonine kinases, which are activated at cell cycle checkpoints, mostly at G0, G1 and S; the DSBs are repaired by two main processes: homologous recombination (HR) and non-homologous recombination end-joining (NHEJ) [73]. A plethora of proteins are used by the cell to protect the genomic stability and the truncated telomeres through the two recombination mechanisms [73, 74].

This complicated and heterogeneous network of signaling pathways and trans-acting regulatory elements serves to maintain telomere length and respond to occurring changes at telomeres. Recently, the telomere position effect (TPE), imposed by relative shortening of telomeres and the rate of loss affecting nearby genes through telomeric chromatin looping has attracted much attention [75, 76]. This is because the TPE explains how physical changes of the truncated telomeres affect other gene expression through physical mechanisms, very often affecting genes placed more than $10 \mathrm{Mb}$ away from the telomeric region [75]. Additionally, short telomeres themselves may serve as another proximate senescence trigger through DNA repair mechanisms [77]. However, many researchers agree that, apart from the aforementioned mechanisms, the relative length of telomeres and the rate of telomere loss affect the genomic structure at most $[30,53,78,79]$. The cell responds accordingly to all these changes, and depending on its current biochemical status, becomes more receptive to other deleterious processes inside of it.

\section{The role of telomerase in cellular pathophysi- ology}

The negative correlation between the telomerase expression and the onset of most cancers in the human body has been favored by the scientific community for years [6163]. The cancerous cells' replicative immortality stressed by Hanahan and Weinberg has been associated with the hTERT locus activation, and thus the conclusion withdrawn was simple: telomerase expression promotes tumorigenesis in somatic cells [80].

Interestingly, the remaining cancer types that do not activate the hTERT locus still elongate their telomeres through the alternative lengthening of telomeres (ALT) mechanism [81, 82]. This route of telomere elongation takes advantage of chromosomal instability and strands breaks, which is detrimental for the cell karyotype. A tumorigenic clone line will always sort the cells with longer telomeres compensating the higher proliferative rate [81]; furthermore, the telomere sister chromatic exchange (TSCE), telomere-repeat arrays exchange resulting from the ALT pathways, yields the unequal sister telomere lengths in different cancerous cell lines [83].

The misconception is that long telomeres are a biomarker for high cancer risk; this is far from the truth. In fact, in most cancerous cells the telomeres are much shorter than those found in normal cells [84, 85]. Most recent tumor studies find that telomeres in the cancer cells are, through structural analysis, truncated and recombinogenic: dicentric chromosomes, reciprocal translocations and other deleterious karyotypes [86, 87]. Moreover, in most studies correlating the telomere length with concomitant cancer, the telomere length has either been found normal or reduced. In other studies, the telomere length has been 


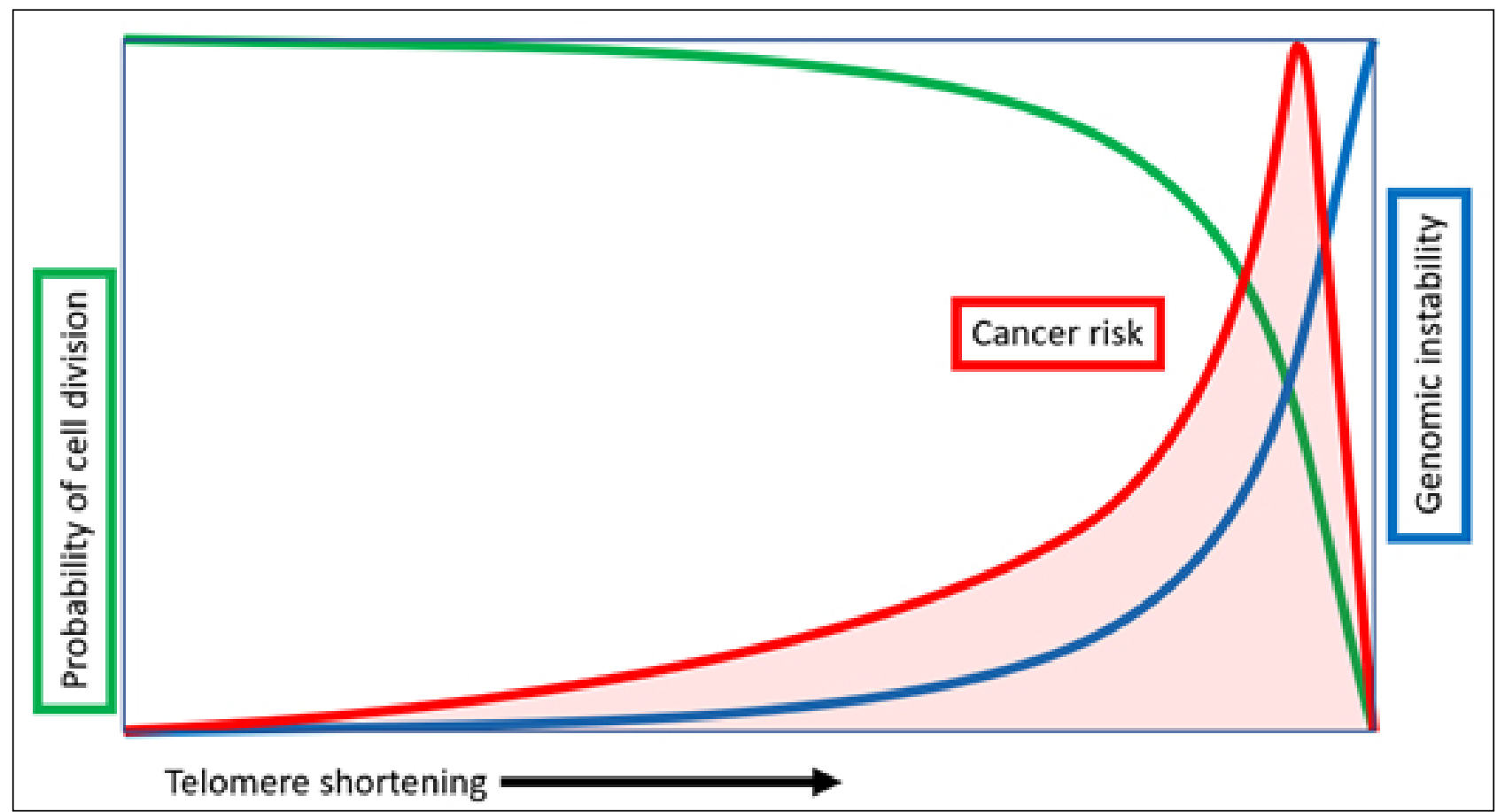

Figure 3. Relationship between telomere shortening, increasing genomic instability and varying cancer risk. With each replicative division the total telomere length decreases, which leads to miscellaneous genomic and intracellular alterations increasing the overall risk of hyperproliferation and tumorigenic mechanisms development. It must be noted that a cell in order to develop cancerous changes must rely on relatively long telomeres for proper genomic stability. If its telomeres are too short, the cell drifts into senescence and eventually dies. Figure created by Michael Fossel, MD, PhD.

found increased with either active or quiescent telomerase [88-91]. In many papers studying the correlation between telomeres length, telomerase expression and cancer cell biology, the histopathological changes in cancer tissue are not reported. Not only is the average length of the cell telomeres measured instead of the shortest telomere length, but these telomere length measurements are taken from the patient's PBLs suffering from a malignant disease that is not correlated with the immune system [30]. These mistakes generate false conclusions and consolidate the misconception of the negative correlation between the telomerase expression and tumorigenesis.

There is much evidence on the relationship between the telomere length maintenance (TLM) and cancer. However, there are no data indicating that cancer is caused by the overexpression of telomerase or the overly long telomeres. Present studies with somatic cells expressing a recombinant telomerase gene or somatic cells treated with telomerase vectors, and studies on the embryonic pluripotent cells with constitutive telomerase expression, indicate no cancer remarks [26, 92-94]. This can be partially explained by the fact that only the average telomere shortening and its rate, not the total telomere loss, determine the senescence phenotype; thus telomerase expression increases genomic stability by keeping the average telomere loss and re-lengthening constant [95]. Simultaneously, the number of cell divisions increases (uncontrolled growth), which leads to further telomere truncation which must be overcome by a higher rate of telomerase expression (Figure 3). These findings can be supported by showing that knocking down the telomerase gene coincides with tumor growth arrest, but only when the tumor had already grown, not before the tumorigenesis process, not even in a p53-null cell [79].

However, telomerase expression does not serve its canonical function only. Recent findings present multiple non-canonical functions of telomerase, which influence genomic stability, very often resembling the ones found in the ALT mechanisms [96]. All in all, both the telomerase- and ALT-based telomere length maintenance pathways stabilize the already destabilized genome of the cancerous cell. Thereby, it is not about a maximum telomere length that can keep the cancer cell from apoptosis, but about keeping telomere length above the critical threshold, different for each organism. This also explains why tumor cells in mice upregulate the mTERT expression, even though the mural telomeres (approx. $50 \mathrm{~kb}$ ) are much longer than the human's (approx. $15 \mathrm{~kb}$-long in a young human cell) [78]. This notion does not hold true for some individuals, however, if their cellular environment manifests a variety of mutations (genome) and preconditioned disorderliness in biochemical pathways and DNA repair mechanisms (cell); although in these cases the telomerase expression introduces new level of complexity to the unbalanced system rather than becoming the leading cause of tumorigenesis. The studies in mice corroborate the observations that, contrary to the general assumptions, telomerase does not increase the risk of tumor development, but also protects the cells from cancerogenesis [97, 98]. 


\section{The beginnings of telomere-targeted therapies}

The number of publications on telomerase and its different biological effects has immensely increased since the dawn of the 2000s [99]. Since the discovery of telomerase, its impact diverged into two schools of thought. The first school is "pro-TERT," favoring its regenerative function at shortened telomeres, extending the cell's population doubling, and restoring mitotic capacity. The other school is mainly "anti-TERT", which extends to applying knowledge of telomeres, telomerase, and their investment in the cell's homeostatic profile in treating miscellaneous cancerous diseases. In fact, the anti-telomerase therapies have been widely exploited in cancer research in the last three decades.

Anti-telomerase molecules have been chosen by selected biotechnology companies as potential drugs, mostly targeted against cancerous tissues [100-102]. One such pharmaceutical, N3'-P5' thio-phosphoramidate (NPS) oligonucleotide (GRN163) acting as a telomerase antagonist, although primarily shown as a competitive and efficient cancer drug introduced by Geron Corporation, had turned out to deliver no expected results. Interestingly, the antitelomerase therapies seem to impair the findings of the opposite school, i.e. upregulating telomerase levels in the cell for therapeutic reasoning. This only showcases that the situation is far more complicated than the initial findings had indicated.

It is still inconclusive and more research must be done to sincerely state that telomerase itself does not increase the risk of cancer development, but there are more than plain premises helping to conclude the germinal findings about telomerase's role in cancer [103]. It must be highlighted again that it is the optimal telomere length that must be present in a cancerous cell in order to satisfy the unstoppable growth. If the telomeres were too long, they would provide the cell with incontestable genomic stability, thus precluding cancer development [104]. On the other hand, if the average length of the cancerous cell's telomeres was too small, the cell would fall into the senescence route and eventually die through apoptosis $[105,106]$. Therefore, we should expect different clinical results depending on the dosage of anti-telomerase therapy, duration, and the activity of anti-telomerase agents. Perhaps, this sort of cancer treatment would yield positive clinical effects in a short run, but the long-term consequences seen in the patient are difficult to predict, especially if to consider the complexity of the tumorigenesis process and the still uncertain role of telomeres and telomerase in its development.

Thereby, instead of focusing sole attention on cancer in the light of telomerase, we should consider the telomerase therapeutic methods that could potentially help confine age-related and other degenerative diseases. Some agree that telomere extension through the reverse transcription of telomerase complex outweighs the risk of cancer promotion [107].

Transient transfection of somatic cells with telomerase transcript, full or truncated, has shown that 1) a causation relationship exists between the length of telomeres and the expressed levels of telomerase, and 2) the extrinsic delivery of telomerase to the cells restores their function, but does not change their phenotypes [26, 108, 109]. The first proof was presented by Bodnar's laboratory in 1998, when human retinal pigment cells and foreskin fibroblasts were transfected with the hTERT-SV40 construct, and established the laboratory immortal cell lines [11]. Bodnar's work initiated a cascade of future experiments using extrinsic telomerase catalytic subunit as the cells' 'transformer', establishing immortalized and phenotypically regular transformed cells [110-112]. The groundbreaking experiment presented by DePinho's lab using a special mural TERT transcript engineered with a knock-in TERTestrogen receptor (mTERT-ER) allele controlled by an extrinsic estrogen receptor modulator, 4-hydroxytamoxifen (4-OHT) showed how reversing the systemic degenerative phenotypes and restoring telomere length in mice with various telomere dysfunctions can be achieved by external, dosage-dependent telomerase expression regulation [92]. Other studies with somatic cells successfully transfected with TERT subunit are reviewed in [107].

From time to time, different therapeutic variations concerning telomerase are reinforced by other biomolecules. In one such study, telomerase therapy was supported by ectopic Bcl-2 transfection [113]. Telomerase as a biomolecule in tissue engineering and regenerative medicine (TERM) has already been strongly suggested [114, 115]. Moreover, it is commonly agreed that transient, ectopic delivery of telomerase under constant physiological control is the only acceptable way for a therapeutic approach. This model, aided by viral vector technology, had been well-tested in the past $[50,116,117]$. The same model, but modified and extended, works perfectly fine with adenoassociated virus (AAV) as a viral vector for ectopic TERT delivery, which does not cause tumor transformation or any genomic instabilities within the transfected cells [93]. Those advancements show that we are ready to embrace the experimental data coming from the telomere research and apply this knowledge to clinics, where the patients could benefit from the billions of dollars already invested in the telomerase, cancer, and gene therapy research.

\section{The future of ageing: Telomerase therapy aims}

Telomerase therapy is rooted in the molecular foundations of cellular pathology. As such, it might target multiple potential diseases directly associated with genomic instability and/or TLM disruption. Every age-related disorder with an etiology correlated with telomere shortening, supported by experimental and clinical data, can become a potential target for therapy. The therapy may not only be used to cure age-related diseases, but it also can be applied to prevent their occurrence. The premise is clear: if we can suppress genomic instability and strengthen cellular homeostasis, the risks of disease onset and its progres- 
sion might be reduced.

Previously in the text, reversing cellular senescence was mentioned. This is an important aspect of every biogerontological therapy which must be addressed with high prudence. From a biomedical standpoint, we are currently not able to reverse molecular ageing in terms of the simple understanding: one cannot reverse the biological clock as this would stand against the second law of thermodynamics. However, one could help suppress the somatic damage to the genome and prevent further deleterious changes happening at the molecular level. These actions will reverse the molecular hallmarks of cellular senescence, giving an individual a better quality of living.

Moreover, as it was already discussed, a strong correlation between cancerous transformation and the telomerase apparatus control exists. If the total loss of telomeric repeats in a given set of chromosomes dictates further mechanisms being activated and deactivated during rigorous checkpoints of the cell cycle, preventing the cell from telomere truncation would yield broadly positive clinical outcomes for cancer. More experimental data is needed to draw proper conclusions, and it is still too early to declare any final statement on the correlation between cancer development and telomere length control, but based on the data we have at the moment we can conclude that there is a promising platform for telomerase therapy targeting cancerous changes at the molecular level. In such terms, telomerase-based gene therapy could become a splendid showcase of the potential of telomerase: to cure age-related disorders and to protect from cancer using one single therapeutic platform.

\section{Evaluating telomerase therapy}

Human clinical studies must be completed before we can state that the therapy is successful and rational. Predictions set a new course of developing gene therapy for age-related diseases, where a biopsychosocial model of disorder must be evaluated for the full understanding of potential telomerase therapy. Telocyte, an American biotechnology company, is one of the first commercial entities focused on producing an effective telomerase-based gene therapy platform to cure Alzheimer's disease and other age-related diseases. As discussed thoroughly by Fossel in his recent publication on the role of telomeres in developing Alzheimer's disease and other dementias, even the ARDs of the brain, with the main functional cells, neurons, mitotically inactive and fully differentiated, can still be explained on the basis of gradually shortening telomeres by taking into account the remaining $80 \%$ of the brain's glial cells which are capable of mitotic division [52]. Because glial cells are supportive cells, their morphological and physiological deterioration affects the neural cells more than it had been predicted $[118,119]$. If the main upstream cause of age-associated dementias are the senescing glial (supportive) cells, whose deteriorated state stems from genomic instability triggered mainly by short- ening telomeres, in this case the progressive functional failure of neurons and the occurrence of the hallmarks of Alzheimer's disease (tau tangles and $\beta$-amyloid plaques) can be fully understood, pathophysiologically, through the biology of telomeres. Therefore, Telocyte's prototypic treatment system is based on extracellular telomerase transcript delivery. It will be done through the AAV-9 vector injected into the spinal fluid and acting transiently on the glial cells. If successful, this gene therapy approach builds a bigger therapeutic platform which will serve to eventually cure most age-related disorders.

The biology of ageing is a crossroad for miscellaneous fields of medicine and biology, where one must not speak about one cause, one effect or a simplified correlational model. Through the use of the powerful proteomics isolated chromatin segments (PICh) method, it has been found that three cell lines manifesting two types of the TLM: telomerase expression and the ALT, were associated with $\sim 400$ different proteins, sharing 98 proteins in common [118]. Many of those proteins could be associated with different genomic loci, cell cycle stages and chromatin looping effects, but this approximation showcases the great abundance of regulatory elements involved directly or indirectly in the processes of telomere length regulation (TRF1, POT1, Apollo), DNA damage mechanisms (Rad50, BLM, ERCC1, PARP1), chromosome end protection (Ku 70, Ku 80, ATM, ATR, WRN), and many others [119-121]. Large numbers of proteins on the cross-talk of different paths must be counted as well, which only adds on to the complexity of the biology of telomeres.

\section{Conclusions}

Should a therapy based solely on telomerase be trusted? This question requires a definitive answer based only on data; no speculations have room here. However, we are certain that telomerase therapy might become the gold standard for all age-related diseases in the next 10 years. Recent findings (cited elsewhere in the text) undoubtedly showcase the positive role of telomerase in the "old" and the senescent cells, which retrieve their biological functions and resupply the pool of "young" and healthy cells of the tissue. The exact role of telomeres and their length control in cancer development is still debatable, but further steps in clinical research will finally resolve this dilemma.

There is yet much to say in ageing research. New discoveries consistently broaden our understanding of telomere biology and demonstrate its complexity, which is understandable in terms of its highly significant role in homeostasis. It must be noted that, even though many signaling pathways and metabolic processes are involved, the main role of telomeres is to protect the chromosomal ends from degradation and the NHEJ, thus protecting the cell from genomic instability and physiological deferment. If this these changes could be stopped and reversed, and the metabolic environment of the cell stabilized, we should 
do it. We must push the progress of our own findings and apply them in the real world, move outside the laboratory settings right into the clinics, where the patients are the final recipients of what once was started in laboratory.

\section{Declarations}

Authors' contributions: Steve Liebich is the sole author of the article.

Acknowledgements: Dr. Woodring Wright, a phenomenal aging and cancer researcher who passed away in August, 2019: his inspiration will live on. Dr Michael Fossel, a technical and graphical supporter of this article. The author's brother whose love will stand the test of time.

Conflict of interest: The author declares no conflict of interest.

\section{References}

1. Blackburn E H, Gall J G. A tandemly repeated sequence at the termini of the extrachromosomal ribosomal RNA genes in Tetrahymena. Journal of molecular biology, 1978, 120(1): 33-53.

2. Olovnikov A M. A theory of marginotomy: the incomplete copying of template margin in enzymic synthesis of polynucleotides and biological significance of the phenomenon. Journal of theoretical biology, 1973, 41(1): 181-190.

3. Meyne J, Ratliff R L, MoYzIs R K. Conservation of the human telomere sequence (TTAGGG) $n$ among vertebrates. Proceedings of the National Academy of Sciences, 1989, 86(18): 7049-7053.

4. Chong L, Van Steensel B, Broccoli D, et al. A human telomeric protein. Science, 1995, 270(5242): 1663-1667.

5. De Lange, T. (2005). Shelterin: the protein complex that shapes and safeguards human telomeres. Genes \& development, 19(18), 2100-2110.

6. Harley CB, Futcher AB, Greider CW. Telomeres shorten during ageing of human fibroblasts. Nature. 1990; 345: 458.

7. Lindsey J, McGill N I, Lindsey L A, et al. In vivo loss of telomeric repeats with age in humans. Mutation Research/DNAging, 1991, 256(1): 45-48.

8. Allsopp R C, Vaziri H, Patterson C, et al. Telomere length predicts replicative capacity of human fibroblasts. Proceedings of the National Academy of Sciences, 1992, 89(21): 10114-10118.

9. Lombard D B, Chua K F, Mostoslavsky R, et al. DNA repair, genome stability, and aging. Cell, 2005, 120(4): 497512.

10. Greider C W, Blackburn E H. A telomeric sequence in the RNA of Tetrahymena telomerase required for telomere repeat synthesis. Nature, 1989, 337(6205): 331-337.

11. Bodnar A G, Ouellette M, Frolkis M, et al. Extension of life-span by introduction of telomerase into normal hu- man cells. science, 1998, 279(5349): 349-352.

12. Cong Y S, Wright W E, Shay J W. Human telomerase and its regulation. Microbiology and molecular biology reviews, 2002, 66(3): 407-425.

13. Collins K, Mitchell J R. Telomerase in the human organism. Oncogene, 2002, 21(4): 564-579.

14. Shay J W, Wright W E. Telomeres and telomerase in normal and cancer stem cells. FEBS letters, 2010, 584(17): 3819-3825.

15. Hayflick L, Moorhead P S. The serial cultivation of human diploid cell strains. Experimental cell research, 1961, 25(3): 585-621.

16. Medvedev Z A. An attempt at a rational classification of theories of ageing. Biological Reviews, 1990, 65(3): 375398.

17. Jin K. Modern biological theories of aging. Aging and disease, 2010, 1(2): 72.

18. Harraan D. Aging: a theory based on free radical and radiation chemistry. 1955.

19. Berlett B S, Stadtman E R. Protein oxidation in aging, disease, and oxidative stress[J]. Journal of Biological Chemistry, 1997, 272(33): 20313-20316.

20. Cadenas E, Davies K J A. Mitochondrial free radical generation, oxidative stress, and aging. Free radical biology and medicine, 2000, 29(3-4): 222-230.

21. Pham-Huy L A, He H, Pham-Huy C. Free radicals, antioxidants in disease and health. International journal of biomedical science: IJBS, 2008, 4(2): 89.

22. Fossel M. The Telomerase Revolution: The Enzyme That Holds the Key to Human Aging and Will Soon Lead to Longer, Healthier Lives. BenBella Books, Inc., 2015.

23. Blasco M A. Telomere length, stem cells and aging. Nature chemical biology, 2007, 3(10): 640-649.

24. Blackburn E H, Epel E S, Lin J. Human telomere biology: a contributory and interactive factor in aging, disease risks, and protection. Science, 2015, 350(6265): 11931198.

25. Shay J W, Wright W E. Hallmarks of telomeres in ageing research. The Journal of Pathology: A Journal of the Pathological Society of Great Britain and Ireland, 2007, 211(2): 114-123.

26. Morales C P, Holt S E, Ouellette M, et al. Absence of cancer-associated changes in human fibroblasts immortalized with telomerase. Nature genetics, 1999, 21(1): 115118.

27. Faragher R G A, Mulholland B, Tuft S J, et al. Aging and the cornea. British journal of ophthalmology, 1997, 81(10): 814-817.

28. Aikata H, Takaishi H, Kawakami Y, et al. Telomere reduction in human liver tissues with age and chronic inflammation. Experimental cell research, 2000, 256(2): 578582.

29. Fossel M. Cell senescence in human aging: A review of the theory. In Vivo, 2000, 14(1): 29-34.

30. Fossel M. Use of telomere length as a biomarker for aging and age-related disease. Current Translational Geriatrics and Experimental Gerontology Reports, 2012, 1(2): 121-127. 
31. Laberthonnière $\mathrm{C}$, Magdinier $\mathrm{F}$, Robin $\mathrm{J} D$. Bring it to an end: does telomeres size matter? Cells, 2019, 8(1): 30.

32. Calvert P A, Liew T V, Gorenne I, et al. Leukocyte telomere length is associated with high-risk plaques on virtual histology intravascular ultrasound and increased proinflammatory activity. Arteriosclerosis, thrombosis, and vascular biology, 2011, 31(9): 2157-2164.

33. Valdes A M, Andrew T, Gardner J P, et al. Obesity, cigarette smoking, and telomere length in women. The lancet, 2005, 366(9486): 662-664.

34. Shen J, Gammon M D, Terry M B, et al. Telomere length, oxidative damage, antioxidants and breast cancer risk. International journal of cancer, 2009, 124(7): 1637 1643.

35. Starr J M, Shiels P G, Harris S E, et al. Oxidative stress, telomere length and biomarkers of physical aging in a cohort aged 79 years from the 1932 Scottish Mental Survey. Mechanisms of ageing and development, 2008, 129(12): 745-751.

36. Ilmonen P, Kotrschal A, Penn D J. Telomere attrition due to infection. PloS one, 2008, 3(5): e2143.

37. Plunkett F J, Franzese O, Belaramani L L, et al. The impact of telomere erosion on memory CD8+ T cells in patients with X-linked lymphoproliferative syndrome. Mechanisms of ageing and development, 2005, 126(8): 855-865.

38. Effros R B, Allsopp R, Chiu C, et al. Shortened telomeres in the expanded CD28-CD8+ cell subset in HIV disease implicate replicative senescence in HIV pathogenesis. Aids, 1996, 10(8): F17-22.

39. Aviv A, Chen W, Gardner J P, et al. Leukocyte telomere dynamics: longitudinal findings among young adults in the Bogalusa Heart Study. American journal of epidemiology, 2009, 169(3): 323-329.

40. Matsubara Y, Murata M, Yoshida T, et al. Telomere length of normal leukocytes is affected by a functional polymorphism of hTERT. Biochemical and biophysical research communications, 2006, 341(1): 128-131.

41. Nordfjäll K, Osterman P, Melander O, et al. hTERT$1327 \mathrm{~T} / \mathrm{C}$ polymorphism is not associated with age-related telomere attrition in peripheral blood. Biochemical and biophysical research communications, 2007, 358(1): 215-218.

42. Zhang D, Wen X, Zhang L, et al. DNA methylation of human telomerase reverse transcriptase associated with leukocyte telomere length shortening in hyperhomocysteinemia-type hypertension in humans and in a rat model. Circulation Journal, 2014: CJ-14-0233.

43. Brouilette S W, Moore J S, McMahon A D, et al. Telomere length, risk of coronary heart disease, and statin treatment in the West of Scotland Primary Prevention Study: a nested case-control study. The Lancet, 2007, 369(9556): 107-114.

44. Valdes A M, Richards J B, Gardner J P, et al. Telomere length in leukocytes correlates with bone mineral density and is shorter in women with osteoporosis. Osteoporosis International, 2007, 18(9): 1203-1210.

45. Sampson M J, Winterbone M S, Hughes J C, et al. Mono- cyte telomere shortening and oxidative DNA damage in type 2 diabetes. Diabetes care, 2006, 29(2): 283-289.

46. Fitzpatrick A L, Kronmal R A, Kimura M, et al. Leukocyte telomere length and mortality in the Cardiovascular Health Study. Journals of Gerontology Series A: Biomedical Sciences and Medical Sciences, 2011, 66(4): 421-429.

47. Savale L, Chaouat A, Bastuji-Garin S, et al. Shortened telomeres in circulating leukocytes of patients with chronic obstructive pulmonary disease. American journal of respiratory and critical care medicine, 2009, 179(7): 566571.

48. Cronkhite J T, Xing C, Raghu G, et al. Telomere shortening in familial and sporadic pulmonary fibrosis. American journal of respiratory and critical care medicine, 2008, 178(7): 729-737.

49. Scheinberg P, Cooper J N, Sloand E M, et al. Association of telomere length of peripheral blood leukocytes with hematopoietic relapse, malignant transformation, and survival in severe aplastic anemia. Jama, 2010, 304(12): 1358-1364.

50. Funk, W. D., Wang, C. K., Shelton, D. N., Harley, C. B., Pagon, G. D., \& Hoeffler, W. K. (2000). Telomerase expression restores dermal integrity to in vitro-aged fibroblasts in a reconstituted skin model. Experimental cell research, 258(2), 270-278.

51. Fyhrquist F, Saijonmaa 0, Strandberg T. The roles of senescence and telomere shortening in cardiovascular disease. Nature Reviews Cardiology, 2013, 10(5): 274-283.

52. Fossel M. A unified model of dementias and age-related neurodegeneration. Alzheimer's \& Dementia, 2020, 16(2): 365-383.

53. Fossel M. Telomerase and the aging cell: implications for human health. JAMA, 1998, 279(21): 1732-1735.

54. Vaziri H, Dragowska W, Allsopp R C, et al. Evidence for a mitotic clock in human hematopoietic stem cells: loss of telomeric DNA with age. Proceedings of the National Academy of Sciences, 1994, 91(21): 9857-9860.

55. Zhang J, Li L. Stem cell niche: microenvironment and beyond. Journal of Biological Chemistry, 2008, 283(15): 9499-9503.

56. Sharpless N E, DePinho R A. How stem cells age and why this makes us grow old. Nature reviews Molecular cell biology, 2007, 8(9): 703-713.

57. Cong Y S, Wen J, Bacchetti S. The human telomerase catalytic subunit hTERT: organization of the gene and characterization of the promoter. Human molecular genetics, 1999, 8(1): 137-142.

58. Liebich S. hTERT Promoter Regulation by Differentiation Mechanisms vs Telomerase Activity in Somatic, Embryonic, and Cancerous Cells. OBM geriatrics. 2019; 3(2):114.

59. Wright W E, Piatyszek M A, Rainey W E, et al. Telomerase activity in human germline and embryonic tissues and cells. Developmental genetics, 1996, 18(2): 173-179.

60. Liu K, Schoonmaker M M, Levine B L, et al. Constitutive and regulated expression of telomerase reverse transcriptase (hTERT) in human lymphocytes. Proceedings of the National Academy of Sciences, 1999, 96(9): 5147- 
5152.

61. Shay J W, Bacchetti S. A survey of telomerase activity in human cancer. European journal of cancer, 1997, 33(5): 787-791.

62. Kim N W, Piatyszek M A, Prowse K R, et al. Specific association of human telomerase activity with immortal cells and cancer. Science, 1994, 266(5193): 2011-2015.

63. Artandi S E, DePinho R A. Telomeres and telomerase in cancer. Carcinogenesis, 2010, 31(1): 9-18.

64. Liebich S. hTERT Promoter Regulation by Differentiation Mechanisms vs Telomerase Activity in Somatic, Embryonic, and Cancerous Cells. OBM Geriatrics 2019;3(2):14.

65. Ulaner G A, Hu J F, Vu T H, et al. Telomerase activity in human development is regulated by human telomerase reverse transcriptase (hTERT) transcription and by alternate splicing of hTERT transcripts. Cancer research, 1998, 58(18): 4168-4172.

66. Wang J, Xie L Y, Allan S, et al. Myc activates telomerase. Genes \& development, 1998, 12(12): 1769-1774.

67. Xu D, Popov N, Hou M, et al. Switch from Myc/Max to Mad1/Max binding and decrease in histone acetylation at the telomerase reverse transcriptase promoter during differentiation of HL60 cells. Proceedings of the National Academy of Sciences, 2001, 98(7): 3826-3831.

68. Ramlee M K, Wang J, Toh W X, et al. Transcription regulation of the human telomerase reverse transcriptase (hTERT) gene. Genes, 2016, 7(8): 50.

69. Cong Y S, Wright W E, Shay J W. Human telomerase and its regulation. Microbiology and molecular biology reviews, 2002, 66(3): 407-425.

70. Cairney C J, Keith W N. Telomerase redefined: integrated regulation of hTR and hTERT for telomere maintenance and telomerase activity. Biochimie, 2008, 90(1): 13-23.

71. Palm W, de Lange T. How shelterin protects mammalian telomeres. Annual review of genetics, 2008, 42: 301-334.

72. Khanna K K, Jackson S P. DNA double-strand breaks: signaling, repair and the cancer connection. Nature genetics, 2001, 27(3): 247-254.

73. Abraham R T. Cell cycle checkpoint signaling through the ATM and ATR kinases. Genes \& development, 2001, 15(17): 2177-2196.

74. Jackson S P. Sensing and repairing DNA double-strand breaks. Carcinogenesis, 2002, 23(5): 687-696.

75. Kim W, Ludlow A T, Min J, et al. Regulation of the human telomerase gene TERT by telomere position effect-over long distances (TPE-OLD): implications for aging and cancer. PLoS biology, 2016, 14(12): e2000016.

76. Bouwman B A M, de Laat W. Getting the genome in shape: the formation of loops, domains and compartments. Genome biology, 2015, 16(1): 1-9.

77. Zou Y, Sfeir A, Gryaznov S M, et al. Does a sentinel or a subset of short telomeres determine replicative senescence? Molecular biology of the cell, 2004, 15(8): 3709 3718.

78. Vera E, de Jesus B B, Foronda M, et al. The rate of increase of short telomeres predicts longevity in mammals. Cell reports, 2012, 2(4): 732-737.

79. Muñoz-Lorente M A, Martinez P, Tejera A, et al. AAV9- mediated telomerase activation does not accelerate tumorigenesis in the context of oncogenic K-Ras-induced lung cancer. PLoS genetics, 2018, 14(8): e1007562.

80. Hanahan D, Weinberg R A. The hallmarks of cancer Cell 100 (1): 57-70. Find this article online, 2000.

81. Perrem K, Bryan T M, Englezou A, et al. Repression of an alternative mechanism for lengthening of telomeres in somatic cell hybrids[J]. Oncogene, 1999, 18(22): 33833390.

82. Cesare A J, Reddel R R. Alternative lengthening of telomeres: models, mechanisms and implications. Nature reviews genetics, 2010, 11(5): 319-330.

83. Rudd M K, Friedman C, Parghi S S, et al. Elevated rates of sister chromatid exchange at chromosome ends. PLoS Genet, 2007, 3(2): e32.

84. Feldser D M, Greider C W. Short telomeres limit tumor progression in vivo by inducing senescence. Cancer cell, 2007, 11(5): 461-469.

85. Blasco M A. Telomeres and human disease: ageing, cancer and beyond. Nature Reviews Genetics, 2005, 6(8): 611-622.

86. Bolzán A D, Bianchi M S. Telomeres, interstitial telomeric repeat sequences, and chromosomal aberrations. $\mathrm{Mu}$ tation Research/Reviews in Mutation Research, 2006, 612(3): 189-214.

87. Blackburn E H, Szostak J W. The molecular structure of centromeres and telomeres. Annual review of biochemistry, 1984, 53(1): 163-194.

88. Zhan W H, Ma J P, Peng J S, et al. Telomerase activity in gastric cancer and its clinical implications. World journal of gastroenterology, 1999, 5(4): 316.

89. Counter C M, Hirte H W, Bacchetti S, et al. Telomerase activity in human ovarian carcinoma. Proceedings of the National Academy of Sciences, 1994, 91(8): 2900-2904.

90. Martins C S, Santana-Lemos B A, Saggioro F P, et al. Telomere length and telomerase expression in pituitary tumors. Journal of endocrinological investigation, 2015, 38(11): 1243-1246.

91. Scheinberg P, Cooper J N, Sloand E M, et al. Association of telomere length of peripheral blood leukocytes with hematopoietic relapse, malignant transformation, and survival in severe aplastic anemia. Jama, 2010, 304(12): 1358-1364.

92. Jaskelioff M, Muller F L, Paik J H, et al. Telomerase reactivation reverses tissue degeneration in aged telomerasedeficient mice. Nature, 2011, 469(7328): 102-106.

93. Bernardes de Jesus B, Vera E, Schneeberger K, et al. Telomerase gene therapy in adult and old mice delays aging and increases longevity without increasing cancer. EMBO molecular medicine, 2012, 4(8): 691-704.

94. Jiang X R, Jimenez G, Chang E, et al. Telomerase expression in human somatic cells does not induce changes associated with a transformed phenotype. Nature genetics, 1999, 21(1): 111-114.

95. Counter C M, Avilion A A, LeFeuvre C E, et al. Telomere shortening associated with chromosome instability is arrested in immortal cells which express telomerase activity. The EMBO journal, 1992, 11(5): 1921-1929. 
96. Park J I, Venteicher A S, Hong J Y, et al. Telomerase modulates Wnt signalling by association with target gene chromatin. Nature, 2009, 460(7251): 66-72.

97. Varela E, Munoz-Lorente M A, Tejera A M, et al. Generation of mice with longer and better preserved telomeres in the absence of genetic manipulations. Nature communications, 2016, 7(1): 1-16.

98. Tomás-Loba A, Flores I, Fernández-Marcos $\mathrm{P}$ J, et al. Telomerase reverse transcriptase delays aging in cancerresistant mice. Cell, 2008, 135(4): 609-622.

99. Corey D R. Telomeres and telomerase: from discovery to clinical trials. Chemistry \& biology, 2009, 16(12): 12191223.

100.Shea-Herbert B, Pongracz K, Shay J W, et al. Oligonucleotide $\mathrm{N}^{\prime} \rightarrow \mathrm{P} 5^{\prime}$ phosphoramidates as efficient telomerase inhibitors. Oncogene, 2002, 21(4): 638-642.

101.Corey D R. Chemical modification: the key to clinical application of RNA interference? The Journal of clinical investigation, 2007, 117(12): 3615-3622.

102.Asai A, Oshima Y, Yamamoto Y, et al. A novel telomerase template antagonist (GRN163) as a potential anticancer agent. Cancer research, 2003, 63(14): 3931-3939.

103.Harley C B. Telomerase is not an oncogene. Oncogene, 2002, 21(4): 494-502.

104.Murnane J P. Telomere dysfunction and chromosome instability. Mutation research/Fundamental and molecular mechanisms of mutagenesis, 2012, 730(1-2): 28-36.

105.Bernadotte A, Mikhelson V M, Spivak I M. Markers of cellular senescence. Telomere shortening as a marker of cellular senescence. Aging (Albany NY), 2016, 8(1): 3.

106.Herbig U, Jobling W A, Chen B P C, et al. Telomere shortening triggers senescence of human cells through a pathway involving ATM, p53, and p21CIP1, but not p16INK4a. Molecular cell, 2004, 14(4): 501-513.

107.Harley C B. Telomerase therapeutics for degenerative diseases. Current Molecular Medicine, 2005, 5(2): 205-211.

108. Poh M, Boyer M, Solan A, et al. Blood vessels engineered from human cells. The Lancet, 2005, 365(9477): 21222124.

109.Klinger R Y, Blum J L, Hearn B, et al. Relevance and safety of telomerase for human tissue engineering. Proceedings of the National Academy of Sciences, 2006, 103(8):
2500-2505.

110.Steinert S, Shay J W, Wright W E. Transient expression of human telomerase extends the life span of normal human fibroblasts. Biochemical and biophysical research communications, 2000, 273(3): 1095-1098.

111.Wyllie F S, Jones C J, Skinner J W, et al. Telomerase prevents the accelerated cell ageing of Werner syndrome fibroblasts. Nature genetics, 2000, 24(1): 16-17.

112.Condon J, Yin S, Mayhew B, et al. Telomerase immortalization of human myometrial cells. Biology of reproduction, 2002, 67(2): 506-514.

113.Petersen T, Niklason L. Cellular lifespan and regenerative medicine. Biomaterials, 2007, 28(26): 3751-3756.

114.Shay J W, Wright W E. Use of telomerase to create bioengineered tissues. Annals of the New York Academy of Sciences, 2005, 1057(1): 479-491.

115.Jäger $\mathrm{K}$, Walter $\mathrm{M}$. Therapeutic targeting of telomerase. Genes, 2016, 7(7): 39.

116.Murasawa S, Llevadot J, Silver M, et al. Constitutive human telomerase reverse transcriptase expression enhances regenerative properties of endothelial progenitor cells. circulation, 2002, 106(9): 1133-1139.

117.Verra N C V, Jorritsma A, Weijer K, et al. Human telomerase reverse transcriptase-transduced human cytotoxic $\mathrm{T}$ cells suppress the growth of human melanoma in immunodeficient mice. Cancer Research, 2004, 64(6): 21532161.

118.Boccardi V, Pelini L, Ercolani S, et al. From cellular senescence to Alzheimer's disease: The role of telomere shortening. Ageing research reviews, 2015, 22: 1-8.

119. Flanary B E, Sammons N W, Nguyen C, et al. Evidence that aging and amyloid promote microglial cell senescence. Rejuvenation research, 2007, 10(1): 61-74.

120.Déjardin J, Kingston R E. Purification of proteins associated with specific genomic Loci. Cell, 2009, 136(1): 175186.

121.De Boeck G, Forsyth R G, Praet M, et al. Telomere-associated proteins: cross-talk between telomere maintenance and telomere-lengthening mechanisms. The Journal of Pathology: A Journal of the Pathological Society of Great Britain and Ireland, 2009, 217(3): 327-344.

Cite this article as: Liebich $\mathrm{S}$. The cellular senescence unification model and telomerase therapy: To treat all age-related diseases[J]. Aging Pathobiology and Therapeutics, 2020, 2(3): 143-154. 